\title{
T-ray Sensing Applications: Review of Global Developments
}

\author{
Tamath Rainsford ${ }^{a}$, Samuel P. Mickan ${ }^{a}$, and Derek Abbott $^{a}$ \\ ${ }^{a}$ Centre for Biomedical Engineering and \\ School of Electrical and Electronic Engineering, \\ The University of Adelaide, SA 5005, Australia.
}

\begin{abstract}
Terahertz wavelengths can pass through dry, non-polar, non-metallic materials that are opaque at visible wavelengths. Moreover they can be manipulated using millimeter wave and quasi-optical techniques to form an image. Sensing in this band potentially provides advantages in a number of areas of interest for security and defense, such as screening of personnel for hidden objects, and the detection of chemical and biological agents. This paper reviews recent research into THz applications by groups across Europe, the US, Australasia, and the UK. Several private companies are developing smaller and cheaper reliable devices allowing for commercialisation of these applications. While there are a number of challenges to be overcome there is little doubt that $\mathrm{THz}$ technologies will play a major role in the near future for advancement of security, public health and defense.
\end{abstract}

Keywords: terahertz, THz, terahertz imaging, terahertz spectroscopy, T-rays

\section{INTRODUCTION}

The 'terahertz gap' that lies between the infrared and millimeter regions of the electromagnetic spectrum (Fig. 1) has only recently become experimentally available. Terahertz ( $\mathrm{THz}$ ) waves, or T-rays, bridge the gap between

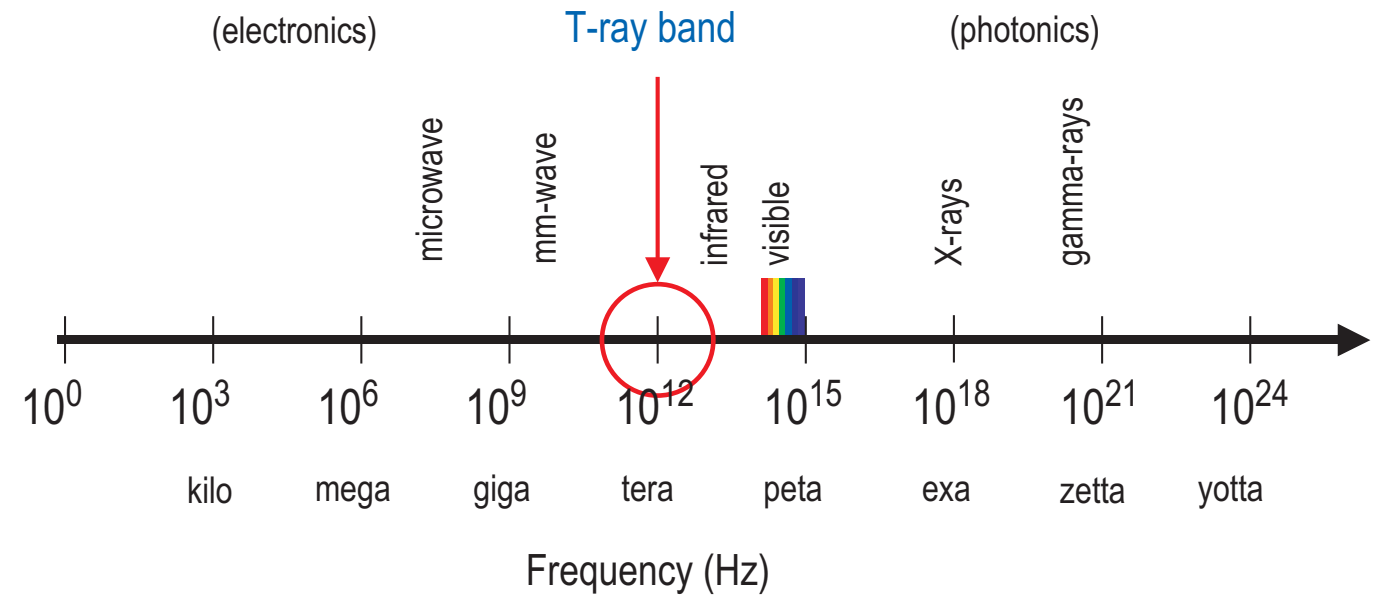

Figure 1: A representation of the electromagnetic spectrum, showing the T-ray band between the well-developed fields of millimeter waves (mm-waves) and the infrared. The T-ray band lies between 0.1 and 10 terahertz (THz).

electronics and photonics, have novel properties and interact uniquely with many materials. The interest in $\mathrm{THz}$ was spawned both by researchers utilising the microwave end of the spectrum and wanting to work with shorter wavelengths, and researchers at the infrared end who saw the need for working with longer wavelengths.

Send correspondence to Tamath Rainsford

E-mail: tamath@eleceng.adelaide.edu.au, Telephone: +61 883036393

E-mail: samuel.mickan@adelaide.edu.au, Telephone: +61 883034115

E-mail: dabbott@eleceng.adelaide.edu.au, Telephone: +61 883035748 
Originally, T-rays were generated and detected by employing conventional techniques borrowed from microwave and millimeter technologies. Since the advent of solid-state Continuous Wave (CW) and ultrafast pulsed lasers, used with biased semiconductors and nonlinear crystals, there have been significant advances in THz technologies. The rapid development of such technologies has allowed researchers to shift their focus to the applications, which are numerous and as yet under-exploited.

$\mathrm{THz}$ sensing was first, and still is, applied in the areas of astronomy and atmospheric science. ${ }^{1}$ In fact, imaging techniques that have grown out of non-space applications are often transferred to areas such as planetary and cometary sensing, as well as earth-based studies including the monitoring of ozone depletion. As there is an increasing military dependence on space-related systems it is likely that $\mathrm{THz}$ radiation will play a major role in the development of future space capabilities such as satellite to satellite communications.

Various rotational, vibrational and translational modes of molecules are within the THz range (0.1-10 THz). Since these modes are unique to a particular molecule it is possible to obtain a "THz fingerprint" allowing for the identification of chemical substances. Astronomers and chemists have already utilised $\mathrm{THz}$ to characterise and identify many small organic molecules. The same techniques can be diversely applied, for example, in the detection of anthrax providing novel approaches for counter-terrorism. THz spectroscopy allows not only for exploration of molecular structures but of molecular dynamics. ${ }^{2}$ Since the structure of biomolecules is closely related to their functionality there are broad ranging applications in biomedicine, for example in DNA sensing. ${ }^{3}$ The wavelength of T-rays makes them a potentially useful carrier medium in secure and short-range line-of-sight communications. The wavelength being short enough to provide significantly higher bandwidth than microwaves, and yet long enough to provide greatly reduced Rayleigh scattering over infrared. The reduced Rayleigh scattering suggests applications in short-range battlefield communications, where smoke and aerosols would block infrared transmission. $\mathrm{THz}$ has low photon energies compared with X-rays, thus it is not considered intrinsically harmful to living tissue. ${ }^{4}$ In addition, it has the ability to penetrate a few millimetres of skin. Hence diagnostics of subcutaneous tissue, such as the detection of skin cancer in vivo, is possible. ${ }^{5}$

T-rays are also able to penetrate dielectric materials, something that cannot be done effectively outside this band. This allows for the detection of chemical and biological agents, and the remote imaging of personnel to detect hidden plastic and metal objects. Sensing in this band potentially provides advantages in a number of areas of interest to security and defense such as short-range radar sensing, as $\mathrm{THz}$ can better penetrate through fog than optical light. The lower scattering of $\mathrm{THz}$ means significantly better imaging than with IR. In medical transillumination applications, for instance, much higher contrast can be achieved than with light or X-rays. As they are transparent to THz, tomographical images of materials, such as ceramics, can be made. ${ }^{6,7}$ This is carried out using time-delay detection of reflected $\mathrm{THz}$ pulses from the surface of the structure under investigation. ${ }^{8,9}$ These techniques have applications in industry for quality control, as surface defects, contaminants and can be detected cost-efficiently.

To date, technology has tended to dictate the applications to explore. More recently, though, the applications themselves have impacted upon technology development. For many of the $\mathrm{THz}$ applications to become an everyday reality, low-cost compact $\mathrm{THz}$ sources and detectors will be required. This means further advances will need to be made in the areas of electronics and nanotechnology.

Across Europe, the US, Australia and Japan, a number of research groups at universities and in industry are working on $\mathrm{THz}$ and ultrafast science and technologies. Funding for T-ray technology mainly comes from the National Science Foundation, the National Institute of Health and the US Departments of Energy and Defense and also, the European Union. Indeed, in the last few years the U.S. Army and the Department of Defense have focused on the advancements of THz-frequency electronic technology and on novel applications of THz-frequency sensing. A number of private companies are developing smaller, reliable and cheaper devices.

This paper gives an overview of $\mathrm{THz}$ applications and provides details on some representative work currently being undertaken in the areas of biomedicine, security, communication and quality control. It also describes challenges that relate to the commercialisation of some of this T-ray research. While this paper does not cover T-ray generation and detection technology in detail, it provides a brief summary of the current developments in novel materials and nanotechnologies and considers how these contribute to the advancement of next-generation miniaturised and cost-effective $\mathrm{THz}$ applications. 


\section{FAST, NON-INVASIVE AND LOW-COST BIOMEDICAL SENSING}

Biomedical sensing is perhaps the most rapidly developing of all $\mathrm{THz}$ applications, ${ }^{10}$ pervading almost every area of biomedicine and driving technology development toward more cost-effective and readily useable, transportable devices for $\mathrm{THz}$ generation and detection. Currently huge efforts are being made to produce all-solid-state THz sources and opportunities for commercialisation abound.

Spectroscopy was among the first applications of $\mathrm{THz}$ technology, for instance, in the development of basic $\mathrm{THz}$ fingerprints of simple molecules, such as water, carbon monoxide and ozone. ${ }^{2}$ However, it soon was realised that T-rays can probe a range of energy transitions that correspond to the excitation of rotational, vibrational and translational modes in complex organic molecules, including bio-molecules. ${ }^{11}$ In THz absorption spectroscopy the structure, and potentially the dynamics, of complex molecules such as proteins, DNA, ${ }^{12}$ RNA, and larger bio-structures, such as cell clusters and bacterial spores can be studied and analysed. This application of T-rays opens possibilities for fast DNA analysis — in both areas of disease detection and forensics. THz imaging of pathogens such as anthrax is also possible. Bio-surveillance and chemo-surveillance will become increasingly important to protect against biological and chemical attacks.

Since $\mathrm{THz}$ radiation is non-ionising, it has many potential medical applications. Apart from spectroscopic characterisation, T-rays can also provide X-ray-like density images, without depositing harmful amounts of energy in human soft tissue. In fact, T-rays could replace X-rays in dental cavity detection, because they can identify tooth decay in early, remedial stages. ${ }^{13}$ X-rays, on the other hand, detect erosion only once a cavity has formed. Owing to their capacity to penetrate the uppermost layers of skin, T-rays show promise in the early detection of skin cancers, monitoring the progress of wound healing underneath protective opaque dressing, or examining the skin of animals underneath their furs.

Breathing modes of DNA — caused by the stretching of hydrogen bonds between the two DNA strands - vibrations, twisting and global stretching modes are excited by radiation in the $0.1-10 \mathrm{THz}$ band. Thus $\mathrm{THz}$ spectroscopy provides information about DNA structure and dynamics. In DNA analysis, THz absorption spectroscopy can be used, for instance, to discriminate A and B configurations. Additionally, THz spectra differ for DNA from different organisms, ${ }^{14}, 15$ for various single mutations, ${ }^{11}$ for intermediate products during chemical reactions, ${ }^{16}$ and for hybridised versus denatured DNA. ${ }^{3,17}$

DNA-to-DNA hybridisation is a technique used to determine the genetic similarity of DNA sequences and can help to identify genetic disease. Hybridisation is typically detected through the use or fluorescent dyelabelling. Unfortunately this is both costly and time consuming and may even cause modifications to the strand conformation. Another drawback is that fluorescent labels glow non-uniformly, giving rise to detection errors. A number of researchers have thus started to use T-rays to detect hybridisation. ${ }^{3,12,18}$ For label-free detection Brucherseifer et $a l^{3}$ have exploited the dependence of the binding state of DNA on its dielectric properties at $\mathrm{THz}$ frequencies. Their technique involves the preparation of thin homogeneous films of DNA deposited on sapphire substrates. Significantly higher refractive indexes are obtained for the hybridised DNA compared with the denatured DNA. Thin films prevent the DNA from reorganising itself into macroscopic structures, which could potentially lead to intrinsic material responses. Homogeneity is also important in order to avoid resonant scattering effects. A drawback of the technique is that very large amounts of DNA are required since the sample needs to be of a thickness that allows significant interaction with the $\mathrm{THz}$ radiation. Bolivar et al ${ }^{17}$ overcome these problems with a unique wave-guided approach. Thin-film microstrip-lines guide THz signals in plane along the thin films of DNA deposited on the sensing circuits. A passive resonator significantly amplifies the DNA$\mathrm{THz}$ interaction. This approach results in improved sensitivity and a drop of three orders in magnitude in the requirement for DNA material. More recently Nagel et a ${ }^{19}$ have developed a THz biochip capable of conducting several hybridisation experiments on a single chip. Their development further reduces the wasting of material and paves the way for high-throughput THz-biochips.

There are a number of difficulties to be overcome with DNA sensing. The spectra of proteins and DNA can look different at different temperatures ${ }^{11,20,21}$ and depend on the thickness of the DNA sample and the orientation of the strands. To date most work has been done on dry solid film, i.e. under unnatural conditions. This makes practical applications difficult. Thus it would be preferable to perform DNA sensing in vitro. However, there are at least two major obstacles to overcome. Firstly, it is extremely hard to orientate the strands under aqueous 
conditions. Secondly, water is strongly dielectric, whereas large biological molecules are only weakly so. Thus highly sensitive differential methods are required to make DNA sensing in vitro possible. These two obstacles are some of the likely reasons why the progression of biological reactions has not as yet been studied in terahertz spectroscopy.

The most successful optical biosensor in the market place is based on Surface Plasmon Resonance (SPR). ${ }^{22,23}$ A SPR system monitors the binding of molecules to a sensor by probing refractive index changes of the target layer relying on the principle of bioaffinity, that is the affinity between two biomolecules. Bioaffinity can be used in a biosensor by attaching a layer of sensor molecules, or analytes, to a sensor slide, as shown in Fig. 2 . The biosensor slide is used by exposing it to a mixture of unknown molecules, to test if any target molecules, or ligands, are present. SPR detects this new layer by probing the refractive index change with an optical laser beam - a change in refractive index results in a change in the intensity of light reflected off the film at some angle, given by the experiment. SPR relies on the refractive index change at optical wavelengths, as determined by the laser. Certain biomolecules, however, do not show contrast at optical wavelengths. Due to the absorption resonances, such molecules could be sensed using T-rays. A new detection method based on T-rays operating on

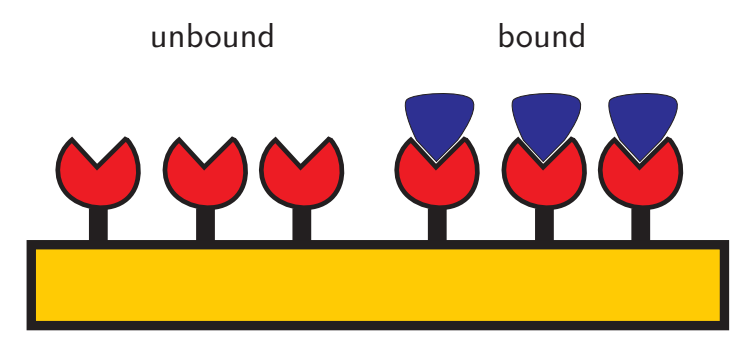

Figure 2: This sketch shows a slide with chemically attached sensor molecules, for example Avidin. The sensor molecules have a strong binding affinity for a target molecule, for example Biotin, which creates a second layer of material when it binds to the sensors. Other molecules that do not bind to the sensor will be washed away and not form an extra layer. The ligand is chosen to have a high binding affinity and high specificity (that is, it will not bind to non-target molecules) for the analyte.

the same principle as SPR has been proposed, and used successfully to detect minute amounts of label-free DNA molecules. $^{24}$ This approach uses integrated T-ray waveguides, incorporating resonant T-ray structures, and has achieved femtomole (fm) sensitivity to label-free DNA hybridisation using T-rays. ${ }^{25,26}$ This approach opens up new avenues for label-free detection. However, the integrated waveguides require application-dependent hardware modifications, delicate sample handling procedures, and the interconnection of a large number of resonators for simultaneous detection.

Mickan et $a l^{27}$ have carried out preliminary testing of the sensitivity of the T-ray DTDS spectrometer to thin layers of bound biomolecules. T-ray signals were observed for Biotin molecules bound to an Avidin sensor, Avidin molecules bound to a Biotin sensor, bead-enhanced Avidin bound to Biotin, and the explosive trinitrotoluene (TNT) bound to TNT antibodies.

Avidin and Biotin together constitute a pair of biomolecules commonly used in demonstrations of bioaffinity, due to their high binding affinity. Bead-enhanced Avidin target molecules create a much thicker molecular layer than Avidin target molecules alone. Avidin is enhanced by binding to large, $\mu \mathrm{m}$-sized Agarose beads. Agarose is an inert substance, extracted from Agar, that is easy to derivitise, or attach to other biomolecules. The Agarose beads attached to the Avidin amplify the target layer thickness, potentially increasing the biosensor's signal.

The protein Avidin was chosen to test the T-ray biosensor due to its high binding affinity to the lipid Biotin and its usefulness in other applications. Avidin comprises four identical subunits, each of which binds one Biotin molecule - see Fig. 3. The binding affinity between Avidin and Biotin is so high $\left(K_{a}=10^{15}\right.$ $\mathrm{mol}^{-1}$ ) that the formation of this complex can be regarded as nearly irreversible, on a scale comparable to a covalent bonding. ${ }^{28}$ The high binding affinity of this system has found many applications, for example in 
affinity chromatography, in attaching antibodies to solid surfaces, precipitating liposomes, or targeting cells with liposomes. ${ }^{29,30}$ Importantly, it has been shown that Biotin can be adsorbed to a hydrophobic surface without losing its specificity towards Avidin, leading to the possibility of studying ligand-analyte interactions on supported lipid membranes.

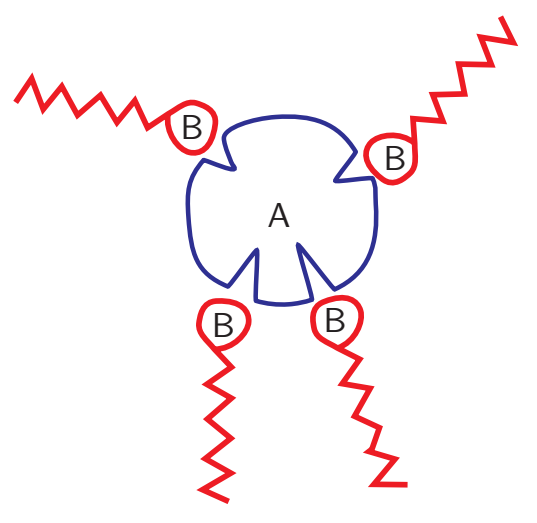

Figure 3: This sketch shows sketches of the molecules Biotin (marked 'B') and Avidin (marked 'A'). The essential characteristics of Biotin are the polar head and long non-polar tail. Avidin, a protein, has four binding sites for Biotin. The molecules are not drawn to scale.

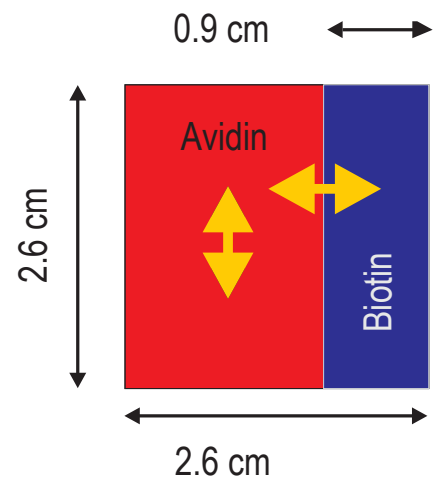

Figure 4: Sample slide for preliminary experiments, showing approximate dimensions in $\mathrm{cm}$. The large doubleheaded arrows approximately indicate the scan range of the T-ray beam across the sample, which was about $10 \mathrm{~mm}$ peak-to-peak. The two cases indicated are Avidin for a test of the signal that occurs due to surface inhomogeneities, and Biotin for a test of bioaffinity sensing. These cases correspond to the waveforms in Fig. 5. 
The biosensor samples were prepared on quartz slides. Quartz is largely transparent to T-rays and biosensor molecules can be chemically attached to its surface. A sketch of the prepared slide is shown in Fig. 4. The T-ray DTDS* waveform measured through the sample and labelled 'Biotin-Avidin' is shown in Fig. 5. The time-domain waveforms are sufficient to distinguish between the bound and unbound states of the biosensor. To estimate the accuracy of the result, and test that the signal was coming from the bound layer of target molecules alone, a DTDS signal was measured from the slide where no Biotin was present, labelled 'pure Avidin' in Fig. 5 and corresponding to the vertical yellow arrow in Fig. 4. The waveforms shown in Fig. 5 indicate that the DTDS signal caused by the presence of a thin layer of target molecules is larger than the signal from the sensor with no target molecules present. This indicates the potential utility of a T-ray-based biosensor.

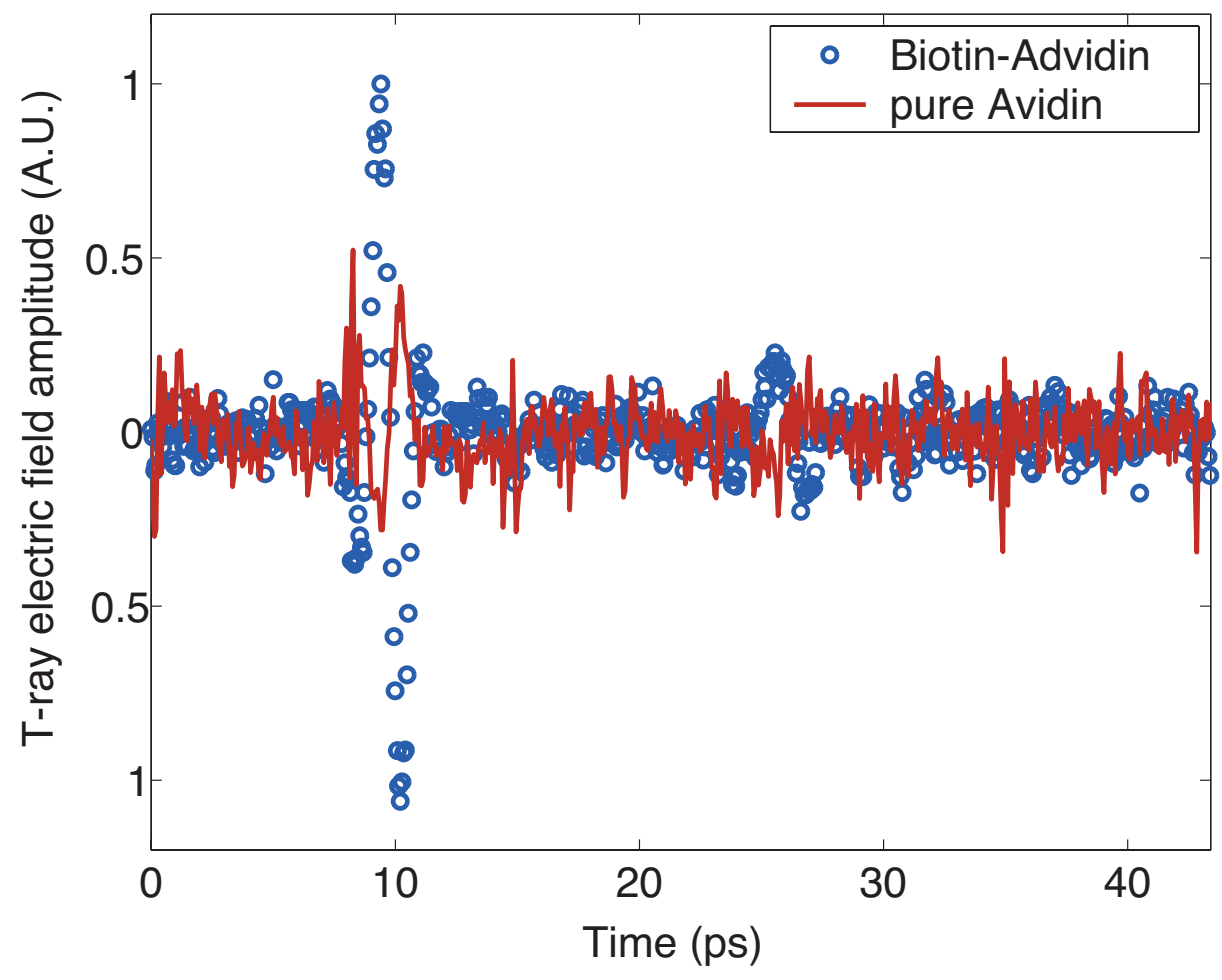

Figure 5: Two time-domain scans are made, one to test for the presence of an Avidin layer ('Biotin-Avidin') and one to confirm that the measurements are due solely to the Avidin layer ('pure Avidin'). The DTDS waveform caused by dithering across the Biotin-Avidin boundary (see Fig. 4) is shown in blue circles. This signal is greater than $2 \times$ the noise level due to surface inhomogeneities in the film, shown in red. The concentration of the Avidin layer is less than $15 \mathrm{ng} / \mathrm{cm}^{2}$. These waveforms were detected with a LIA time constant of $300 \mathrm{~ms}$ and each scan took 15 mins. The spot size of the T-ray beam on the sample is $5 \mathrm{~mm}$, limited by a metal iris placed in the beam path less than $5 \mathrm{~mm}$ from the sample (99\% of signal transmitted). The SNR is 10 . The secondary pulse is due to a Fabry-Pérot reflection of the T-ray pulse inside the quartz slide.

Currently skin cancer is identified via visual inspection, and biopsies are taken whenever there is doubt. Teraview, a company based in Cambridge, UK, was established in 2001 to develop and exploit THz technology. Pickwell et al from Cambridge University, in collaboration with Teraview, have shown with both modelling and human THz-pulsed imaging (TPI), ${ }^{5}$ that T-rays can differentiate between normal and cancerous tissue. Although only able to penetrate a few millimetres of tissue, $85 \%$ of all cancers lie in the epithelium, allowing $\mathrm{THz}$ to sense, in vivo, very small size cancers such as basal cell carcinomas (BCC). The exact mechanism underlying the

\footnotetext{
${ }^{*}$ DTDS stands for Differential Time Domain Spectroscopy ${ }^{31}$
} 
detection is not as yet well understood, however the image contrast in TPI is likely due to the increased water concentration in BCC tissue. ${ }^{32}$ Indeed, PET and MRI support that tumours have increased water content. ${ }^{33,34}$ Pickwell et $a l^{35}$ are trying to determine the underlying contrast mechanism using a finite-difference time-domain model which simulates the interaction of $\mathrm{THz}$ with water. A number of other techniques have been explored for use in pathological tissue diagnosis and are as follows:

- Ultrasound has been utilised to visualise tumours ${ }^{36}$ but has the drawback that it cannot distinguish between benign and malignant tumours. ${ }^{37,38}$

- Near-infrared can differentiate pigment variations but is depth limited.

- High-resolution, real-time microscopy shows cell structure but has limited penetration and involves tissue excision.

Other disorders of the skin such as psoriasis and eczema also can potentially be diagnosed with TPI. ${ }^{32,39}$ Currently, Cambridge Consultants Ltd. and Teraview Ltd. are working together to establish the best market entry strategy for Teraview's advanced breast cancer imaging technology. Teraview is conducting a series of studies with Addenbrooke's Hospital, Cambridge, UK in which healthy and cancerous tissues are imaged via TPI.

Overall, better detection techniques reduce the need for surgical removal of tissue for analysis purposes. Large tumours are often removed in stages in which, after removal, each specimen is histologically examined. T-ray imaging may make possible a single step removal process. This will enable improved detection rates of unhealthy tissue during surgery and should lead to a decrease in the number of repeat surgeries and in morbidity. Thus application of THz-based imaging technologies could result in significant healthcare savings. Low-cost, portable systems will aid in the detection of skin cancer, reducing the need for costly and traumatic surgery. Mittleman et al are currently working on the development of a $\mathrm{THz}$ endoscope with which, through internal-cavity imaging, colon and cervical cancers can be identified.

\section{PRACTICAL SECURITY SCANNING}

T-ray imaging and spectroscopy techniques have significant promise for security, potentially providing a larger range and better tools in the fight against terrorism. Not only are T-rays able to detect and identify biological and chemical material threats, but also hidden metal and plastic devices. Many companies and organizations, such as Thru Vision and Teraview, are putting considerable efforts into detection systems that are low cost and easily transportable or even hand held. Such systems could potentially detect metal and plastic weapons, explosives, contraband and bio-weapons such as anthrax.

The best screening techniques of people at security checkpoints are fast and non-invasive. Teraview has produced the world's first portable THz imagers and spectrometers. By sending short pulses of THz waves at a person and recording the reflected radiation it is possible to image materials hidden close to a persons body or within packaging materials. However, a person appears to be naked, because T-rays penetrate clothing. This potentially has undesired legal (privacy) implications. In order to overcome this problem, Teraview's THz imaging system is frequency-band filtered. It only searches for those specific frequencies at which explosives or metallic objects reflect or absorb when irradiated with T-rays.

Until now T-ray sources have mainly been based on relatively sophisticated ultrafast pulsed lasers. For the last three years Teraview has been working on developing more convenient sources and detectors, and found a number of ways to improve on performance. With Smiths Detection from Hertfordshire, UK, Teraview has developed a hand-held $\mathrm{THz}$ security wand to scan passengers in airport terminals for the characteristic spectral signatures of metals, explosives and other chemicals such as drugs. With the automated detection system being always alert, all that the operative will see is a green light if everything is okay, and a red light if not. Currently, the system generates $\mathrm{THz}$ radiation by shining pulses from a powerful infrared laser onto a crystal of gallium arsenide. The wand is linked to the laser with an optical fibre. Smiths Detection and Teraview are working towards a more compact but equally powerful diode laser that will fit into the wand itself. 
Another company looking at $\mathrm{THz}$ imaging and its applicability to security is Thru Vision at the Space Science and Technology Department, CCLRC Rutherford Appleton Laboratory. It is particularly interested in the detection of concealed metals, ceramics, plastics and liquids, and has built a product demonstrator - the T-scan 2003A - comprising a camera, control electronics and data acquisition, all within a single, compact, portable unit. The T-scan 2003A builds up a $64 \times 128$ pixel image revealing concealed objects. What makes this system unique is that it utilises passive imaging - it does not illuminate the subject with any form of radiation, but simply generates an image of the subject by 'observing' the subject in the THz region.

Since T-rays can see through paper envelopes (Fig. 6) they could also be employed by mail sorters to check for biological and chemical hazards. ${ }^{40}$ T-rays are able to detect anthrax. ${ }^{41}$ In a series of five experiments, van der Weide ${ }^{40}$ prepared sealed envelopes that contained either the harmless talcum powder or Bacillus cereus mimicking the harmful anthrax. T-rays distinguished between the two in all the tests performed.

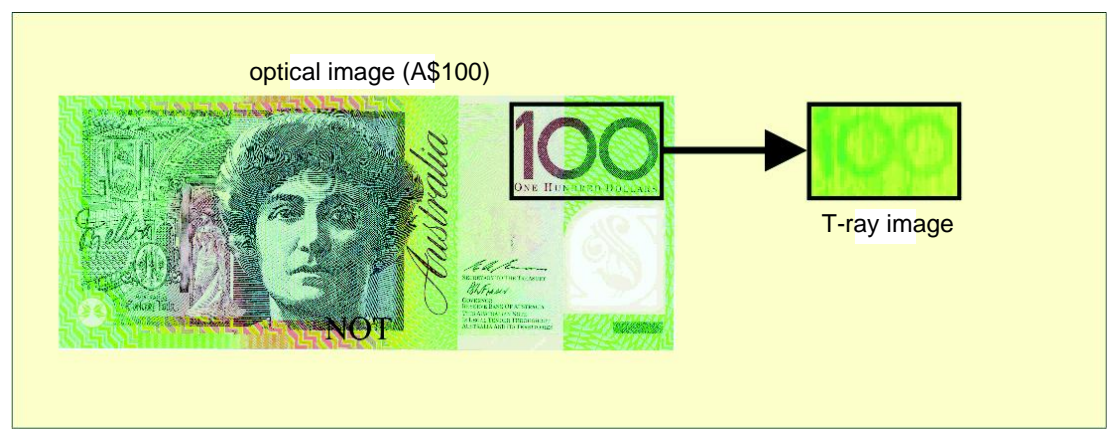

(a) Terahertz transmission image of a portion of an Australian $\$ 100$ note. The T-ray absorption of the ink used to write the '100' is greater than that of the surrounding plastic, so the digits appear darker.

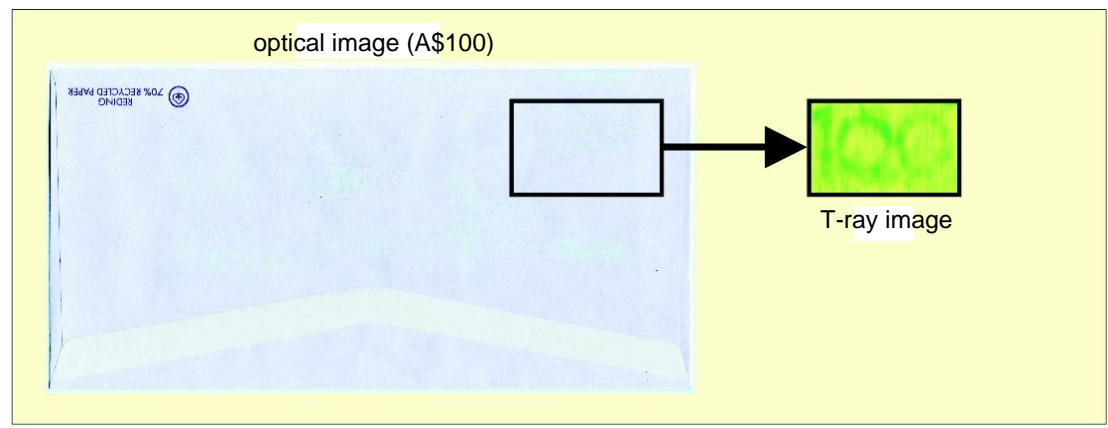

(b) Same note as in Subfig. 6(a), but this time the note is inside a paper envelope. The ' 100 ' still appears because the paper envelope is transparent to T-ray radiation.

Figure 6: This experimental data demonstrates that T-rays can be used to detect contrast in dry, non-polar samples, in this case due to the different T-ray spectroscopic differences in the inks used to print the currency. When the plastic note is placed in an envelope, the writing is no longer visible in the optical image. However, the T-ray image still shows the ' 100 '. The dry paper envelope shows very low T-ray absorption. ${ }^{42}$ The envelope is oriented with the flap at the bottom, not in the T-ray path, otherwise the increased thickness of the flap would be visible in the T-ray image. The image was taken by raster scanning the note in a standard T-ray spectrometer.

There is little doubt that security systems of the near future will incorporate THz technologies. It will be 
increasingly necessary to scan for biological, chemical and other weapons in a manner that is non-invasive and fast. Scanners such as the Smiths Detection/Teraview detection wand and Thru Vision's T-scan 2003A are just the first generation of devices that, in the future, one can expect to find at large events as well as in airports and other secure areas.

\section{INDUSTRIAL SURFACE INSPECTION QUALITY CONTROL AND PROCESS MONITORING}

Quality control is an essential part and parcel of the manufacturing process. Very often industrial surface inspection is undertaken manually, which is tedious, time-consuming, and prone to errors. Innovative techniques in machine vision, combined with novel techniques for quality inspection, can enable automated industrial inspection of components with complex surfaces. This has enormous potential as a cost-effective alternative to manual inspection, and includes the detection of surface defects, such as scratches, erroneous indentations or protrusions and voids that might be present on smooth and textured surfaces of both nominally flat and more complex three-dimensional components. Typical industrial applications of automated quality control include the inspection of ceramic tiles and china products, polished natural stone surfaces, and a range of painted or transfer-printed manufactured components.

There are two major difficulties that need to be resolved in order to make automated inspection more convienient. Firstly, quality control devices need to be incorporated in the production process and thus cannot be placed intimately close to the components to be inspected. Any practical imaging system therefore needs to feature large working distances. Secondly, most manufacturing processes are intrinsically fast. Hence, automated inspection needs to be able to acquire and process data rapidly. T-ray sensing is a promising candidate, potentially able to surmount both of these problems. Because of minimal Rayleigh scattering, THz, unlike IR has a number of advantages for inspection systems. T-rays also have wavelength short enough to be manipulated by optical components, thus $\mathrm{THz}$ images can be analysed quickly, similarly to optical images. Importantly, Hasegawa et $a l^{43}$ have shown that when scanning surfaces with T-rays, they can achieve a high sensitivity to defects despite natural surface roughness. ${ }^{44}$ This is a key finding, as it demonstrates that $\mathrm{THz}$ radiation outperforms visible light, which is scattered significantly by rough surfaces.

Process Analytical Technology (PAT) is the analytical inspection of the manufacturing process throughout the whole procedure. Interestingly, continuous techniques such as those applied in semiconductor processing have not as yet been used in the production of pharmaceuticals. The US Food and Drug Administration have put in place initiatives aimed to accelerate the development of new PATs. THz technology promises to play a major role in these developments. Research has already shown that $\mathrm{THz}$ pulsed spectroscopy can probe the physical state of a pharmaceutically active ingredient. ${ }^{45}$ With T-rays it is also possible to obtain information about potency of paracetamol and aspirin in the presence of other expedients. ${ }^{46}$ Such studies are important because they pave the way for quality-control monitoring at various intermediate steps in the manufacturing process rather than just at the end point. Moreover, because THz spectroscopy can provide information at the molecular level and is able to penetrate blister packets, it can be used to monitor chemical stability and indicate time-related changes in performance.

Inspection of semiconductors - in particular, when resolution at the nanometer scale is required - is another important potential application of T-rays. Researchers at Rensselaer Polytechnic Institute and IMRA America have applied scanning near-field optical microscopy (SNOM) to $\mathrm{THz}$ imaging. ${ }^{47}$ The resolving aperture is replaced by a sharp tip which allows for resolutions down to $150 \mathrm{~nm}$. A dipole moment is induced by $\mathrm{THz}$ radiation in the tip and the region underneath the sampled semiconductor. Scattered radiation is measured, and any remaining signal is detected with a bolometer. Electrooptic sampling allows the electric field of the reflected $\mathrm{THz}$ pulse to be mapped.

A collaboration between the Rensselaer Polytechnic Institute and NASA whose objective is to create a T-ray technology that detects flaws in space shuttle insulation. ${ }^{48}$ The idea is to detect minute, potentially dangerous defects - such as small bubbles and separations from base material — in the foam insulation used on the shuttle's fuel tank. Such faults could have played a role in the 2003 shuttle crash upon re-entry, which was caused by a falling piece of foam that separated from the underlying metal skin during the last of the shuttle's 
launches before hitting the left wing. THz waves are able to penetrate foam to a depth of many inches and thus can potentially scan the whole of the shuttle's fuel tank foam insulation for defects. This is a prime example that highlights exactly where $\mathrm{THz}$ wins over IR and X-ray techniques. In the case of foam, Rayleigh scattering is high causing IR techniques to fail. X-ray imaging also fails, as the photon energy is too high to show good contrast in soft foam.

\section{IMPROVED COMMUNICATION TRANSFER RATES AND VOLUMES}

The full potential of the $\mathrm{THz}$ band for communication has not as yet been realised. Conventional wireless techniques for communication use microwaves at very low power. THz could increase the rate of information transfer as well as the volume. For instance, the Bluetooth ${ }^{T M}$ system currently works at $2.45 \mathrm{GHz}$, however, it is expected that newer systems will operate in the range of 300 to $400 \mathrm{GHz}$. Neil Weste has aptly dubbed this "Bluefang." 49 The goal is to increase operation frequencies of wireless communication into the THz region. One difficulty is that there needs to be line-of-sight connection between emitter and receiver. In order to solve this problem, Koch's group is working on "THz wallpaper." with highly reflective and cheap plastic mirrors, helping to establish an indirect connection. The ultimate vision is secure high-bandwidth Wireless Local Area Networks (WLANs). The advantage of THz over IR for indoor applications is, it is an extremely quiet band without noise or background clutter. For outdoor applications, $\mathrm{THz}$ is expected to play a role in short-range battlefield communications - here the advantage is that $\mathrm{THz}$ will penetrate smoke and aerosols better than IR and will be a more secure higher bandwidth link than microwaves or millimeter waves.

\section{NOVEL MATERIALS AND NANOTECHNOLOGY — THE WAY AHEAD?}

While the $\mathrm{THz}$ frequency regime offers many technical advantages with its wider bandwidth and improved spatial resolution, the solid-state electronics capability within the $\mathrm{THz}$ frequency regime remains extremely limited from a basic signal source and systems perspective. There is a recognised need for developing low-cost, compact and reliable sources of $\mathrm{THz}$ radiation. Research to this end is being done in the areas of nano-devices and systems that utilise nanostructures, nano-processes and nano-integration technology. Such devices will potentially have applications in the information and communications industry as well as in T-ray imaging.

A palm-sized $\mathrm{THz}$ device which exploits the nanostructure of a silicon germanium semiconductor is being developed. The semiconductor is used to select energy bands that relate to $\mathrm{THz}$, and filters control the frequency more precisely. A waveguide allows the signal to be directed in a similar way as in a laser pointer. The device has been used to reveal a paper clip behind a silicon wafer and to scan the interior of an egg through its shell.

Fujimaki et $a l^{51}$ are creating "THz Electronics" based on single-flux-quantum (SFQ) circuits. The idea makes use of high-temperature superconductors with low power consumption and predicts applications such as imaging systems, A/D converters and digital signal processors.

Woodall et al have grown InAs on $\mathrm{GaP} .^{52}$ In this InAs/GaP system the self-annihilation of threading dislocations can cause differential electron mobilities of $20,000 \mathrm{~cm}^{2} /$ Volt/sec for only 2 microns of epilayer growth, thus making it a promising candidate for $\mathrm{THz}$ devices.

Metamaterials are artificially structured materials that extend the properties of existing naturally occurring materials and compounds. In 1968 it was predicted that there might be materials that reverse physical properties. $^{53}$ In 1996, Pendry theorised that certain configurations of metal could have unique responses to electric and magnetic fields. The magnetic activity of natural materials tends to fade away at higher frequencies, making it difficult to sustain magnetism at optical frequencies. Research is well underway to develop a structure that extends the frequency range of magnetic response by more than two orders of magnitude. Smith $e t a l^{54}$ have designed a metamaterial, which responds magnetically in the $\mathrm{THz}$ range, thus opening the potential for a range of $\mathrm{THz}$ applications. 


\section{CONCLUSIONS}

There is little doubt that $\mathrm{THz}$ technologies will play a major role in the future of security, defense and public health. Applications, such as scanning devices for airport terminals that look for specific spectral signatures of chemical and biological threats as well as metal and plastic weapons, are already realised. The focus is now on miniaturising these systems and making them more convenient to use. If a sufficient critical mass of engineering research is funded, we can expect to see $\mathrm{THz}$ screening in practice within a few years. In biomedicine, proof of concept for DNA sensing and the detection of skin cancer and other skin disorders has been reported. Quality control scanning for purity of substances and defects in materials are also established. These techniques need further work and need to become both more economical and more efficient.

While more recent research has focussed on $\mathrm{THz}$ applications, there is still a need to further develop the devices that generate and detect $\mathrm{THz}$ radiation. It is very likely that over the next years these developments will markedly be shaped by progresses in nanotechnology and innovations to come in the creation of new materials. The advances made in aligning nanostructures in semiconductors and superconductors already provide a glimpse of the revolutionary technology that we are likely to see in the near future.

There are a number of challenges on the way ahead, for example, $\mathrm{THz}$ spectroscopic databases need to be created and made available. Also, signal-to-noise ratio in the new $\mathrm{THz}$ devices, acquisition rates and image resolution need to improve. Advancements in these areas will open up biological, security and communication applications even further. However, with a large number of researchers working on applications, generation and detection all over the globe, coming from diverse backgrounds and forming strong collaborations, there is no doubt that in the next decade $\mathrm{THz}$ technologies will advance and spread to new research areas.

\section{ACKNOWLEDGMENTS}

We greatly acknowledge funding from The University of Adelaide and the Sir Ross and Sir Keith Smith Fund.

\section{REFERENCES}

1. D. Turchinovich, A. Kammoun, P. Knobloch, T. Dobbertin, and M. Koch, "Terahetz astronomical telescopes and instrumentation," Phil. Trans. R. Soc. Lond. A 362, pp. 395-402, 2004.

2. F. De Lucia, "Spectroscopy in the terahertz spectral region," in Sensing With Terahertz Radiation, D. Mittleman, ed., pp. 39-115, Springer Verlag, Berlin, 2003.

3. M. Brucherseifer, M. Nagel, P. Bolivar, H. Kurz, A. Bosserhoff, and R. Büttner, "Label-free probing of the binding state of DNA by time-domain THz sensing," Applied Physics Letters 77(24), pp. 4049-4051, 2000.

4. E. Berry, "Risk perception and safety issues," Journal of Biological Physics 29(2-3), pp. 263-267, 2003.

5. R. M. Woodward, B. E. Cole, and V. P. Wallace, "THz pulse imaging of in vitro basal cell carcinoma samples," TOPS 56, pp. 329-330, 2001.

6. M. Brucherseifer, P. H. Bolivar, H. Klingenberg, and H. Kurz, "Angle-dependent THz tomography - characterization of thin ceramic oxide films for fuel cell applications," Applied Physics B - Lasers and Optics 72(3), pp. 361-366, 2001.

7. B. Ferguson, S. Wang, D. Gray, D. Abbott, and X. C. Zhang, "Towards functional 3D T-ray imaging," Physics in Medicine and Biology 47, pp. 3735-3742, 2002.

8. S. Wang and X. C. Zhang, "T-ray computed tomography ", Optics Letters 27(15), pp. 1312-1314, 2004.

9. B. Ferguson, S. H. Wang, D. Gray, D. Abbott, and X. C. Zhang, "Pulsed terahertz tomography," Journal of Physics D - Applied Physics 37(4), pp. R1-R36, 2002.

10. P. H. Siegel, "Terahertz Technology in Biology and Medicine," IEEE Transactions on Microwave Theory and Techniques 52(10), pp. 2438-2447, 2004.

11. A. Markelz, S. Whitmire, J. Hillebrecht, and R. Birge, "THz time domain spectroscopy of biomolecular conformational modes," Physics in Medicine and Biology 47, p. 3797, 2002.

12. A. G. Markelz, A. Roitberg, and E. J. Heilwel, "Pulsed THz spectroscopy of bovine serum albumin and collagen between 0.1 and 2.0THz," Chem. Phys. Lett 320, pp. 42-48, 2000. 
13. D. A. Crawley, C. Longbottom, B. E. Cole, C. M. Ciesla, D. Arnone, V. P. Wallace, and M. Pepper, "Terahertz pulse imaging: A pilot study of potential applications in dentistry," Caries Research 37(5), pp. 352-359, 2003.

14. M. Bykhovskaia, B. Gelmont, T. Globus, D. L. Woolard, A. C. Samuels, T. H. Duong, and K. Zakrzewska, "Prediction of DNA far-IR absorption spectra based on normal mode analysis," Theoretical Chemistry Accounts 106, pp. 22-27, 2001.

15. T. H. Duong and K. Zakrzewska, "Calculation and analysis of low frequency normal modes for DNA," Journal of Computational Chemistry 18(6), pp. 796-811, 1997.

16. R. Beger and E. W. Prohofsky, "Method for calculating the effect of a rigid enzyme on the dynamics of a large DNA helix," Physical Review A 43(10), pp. 5672-5677, 1991.

17. P. Haring-Bolivar, M. Bruchseifer, M. Nagel, H. Kurz, A. Bosserhoff, and R. Büttner, "Label-free probing of genes by time-domain terahertz sensing. Physics in Medicine and Biology," Physics in Medicine and Biology 47, pp. 3815-3821, 2002.

18. D. Woolard, T. Korsica, D. L. Rhodes, H. L. Cui, R. A. Pastore, J. O. Jensen, L. W. R, R. H. Jacobsen, D. Mittlemann, and M. C. Nuss, "Millimeter wave-induced modes in DNA as a possible alternative test to animal tests to probe for carcogenic mutations," J. Appl. Toxicol. 17, p. 243, 1997.

19. M. Nagel, P. Haring Bolivar, and H. Kurz, "A functionalised THz-sensor for marker free DNA-anaysis," Phys. Med. Biol. 48, pp. 3625-3636, 2003.

20. T. R. Globus, D. L. Woolard, A. C. Samuels, B. Gelmont, J. Hesler, T. W. Crowe, and M. Bykhovskaia, "Submillimeter-wave fourier transform spectroscopy of biological macromolecules," Journal of Applied Physics 91(9), pp. 6105-6113, 2002.

21. B. M. Fischer, M. Walther, and P. Uhd Jepsen, "Far infrared vibrational modes of DNA components studied by THz time-domain spectroscopy," Physics in Medicine and Biology 47, pp. 3807-3814, 2002.

22. R. Kalsson and R. Stahlberg Analytical Chemistry 228, p. 274, 1995.

23. X. Song and B. I. Swanson, "Direct, ultrasensitive, and selective optical detection of protein toxins using multivalent interactions," Analytical Chemistry 71(11), pp. 2097-2107, 1999.

24. A. Menikh, R. MacColl, C. A. Mannella, and X.-C. Zhang, "Terahertz biosensing technology: Frontiers and progress," ChemPhysChem 3(8), pp. 655-658, 2002.

25. M. Nagel, P. Haring Bolívar, M. Brucherseifer, H. Kurz, A. Bosserhoff, and R. Büttner, "Integrated THz technology for label-free genetic diagnostics," Applied Physics Letters 80(1), pp. 154-156, 2002.

26. M. Nagel, P. Haring Bolívar, M. Brucherseifer, H. Kurz, A. Bosserhoff, and R. Büttner, "Integrated planar terahertz resonators for femtomolar sensitivity label-free detection of DNA hybridization," Applied Optics 41(10), pp. 2074-2078, 2002.

27. S. P. Mickan, A. Menikh, H. Liu, C. A. Mannella, R. MacColl, D. Abbott, J. Munch, and X. C. Zhang, "Labelfree bioaffinity detection using terahertz technology," Physics in Medicine and Biology 47(21), pp. 3789-3795, 2002.

28. N. M. Green, "Avidin," Advances in Protein Chemistry 29, pp. 85-133, 1975.

29. P. Cuatrecasas and M. Wilchek, "Single-step purification of avidin from egg white by affinity chromatography on biocytin-Sepharose columns," Biochemical and Biophysical Research Communications 33(2), pp. 235-239, 1968.

30. M. Wilchek and E. A. Bayer, "The avidin-biotin complex and bioanalytical applications," Analytical Biochemistry 171(1), pp. 1-32, 1988.

31. S. P. Mickan, D. Abbott, J. Munch, and X.-C. Zhang, "Noise reduction in terahertz thin film measurements using a double modulated differential technique," Fluctuation and Noise Letters 2(1), pp. L205-L220, 2002.

32. R. M. Woodward, B. E. Cole, and V. P. Wallace, "THz pulse imaging in reflection geometry of human skin cancer and skin tissue," Physics in Medicine and Biology 47(21), pp. 3853-3863, 2002.

33. K. F. Ross and R. E. Gordon, "Water in malignant tissue, measured by cell refractometry and nuclear magnetic resonance," J. Microsc. 128, pp. 7-21, 1982.

34. E. K. Rofstad, E. Steinsland, O. Kaalhus, Y. B. Chang, B. Hovik, and H. Lyng, "Magnetic resonance imaging of human melanoma xenografts in vivo : proton spin lattice, spin-spin relaxation times verses fractional water content and fraction of necrotic tissue," Int. J. Radiat. Biol. 65, pp. 387-401, 1994. 
35. E. Pickwell, B. E. Cole, and A. J. Fitzgerald, "In vivo study of human skin using pulsed THz radiation," Physics in Medicine and Biology 49(9), pp. 1595-1607, 2004.

36. N. Lassau, A. Spatz, and M. F. Avril, "Value of high frequency US for preoperative assessment of skin tumors," Radiographics 17, pp. 1559-1565, 1997.

37. B. D. Fornage, M. H. McGavran, M. Duvic, and C. A. Waldron, "Imaging of the skin with 20-MHz US," Radiology 189, pp. 69-76, 1993.

38. K. Hoffmann, M. Happe, and S. Schuller, "Ranking of $20 \mathrm{MHz}$ sonography of malignant melanoma and pigmented lesions in routine diagnosis," Ultraschall Med. 20, pp. 104-109, 1999.

39. B. E. Cole, R. M. Woodward, and D. Crawley, "THz imaging and spectroscopy of human skin, in vivo," Proc. SPIE 4276, pp. 1-10, 2001.

40. M. K. Choi, A. Bettermann, and D. W. van der Wiede, "Potential for detection of explosive and biological hazards with electronic terahertz systems," Phil. Trans. R. Soc. Lond. A 362, pp. 337-349, 2004.

41. B. Ferguson, S. Wang, D. Abbott, and X. C. Zhang, "Powder retection with THz imaging," Proc SPIE Terahertz for Military and Security Applications 5070, pp. 7-16, 2003.

42. S. P. Mickan, D. Abbott, J. Munch, X.-C. Zhang, and T. van Doorn, "Analysis of system trade-offs for terahertz imaging," Microelectronics Journal (Elsevier) 31(7), pp. 503-514, 2000.

43. N. Hasegawa, T. Loffler, M. Thomson, R. Leonhardt, and H. G. Roskos Appl. Phys. Lett 83, pp. 3996-3998, 2003.

44. T. Loffler, K. J. Siebert, H. Quast, N. Hasegawa, G. Loata, R. Wipf, T. Hahn, M. Thomson, R. Leonhardt, and H. G. Roskos, "All-optoelectronic continous-wave THz systems," Phil. Trans. R. Soc. Lond. A 362, pp. 263-281, 2004.

45. P. F. Taday, I. V. Bradley, D. D. Arnone, and M. Pepper, "Using Terahertz pulse spectroscopy to study the crystalline structure of a drug: a case study of the polymorphs of ranitidine hydrochloride," J. Pharm. Sci. 92, pp. 831-838, 2003.

46. P. F. Taday, "Applications of Terahertz spectroscopy to pharmaceutical sciences," Phil. Trans. R. Soc. Lond. A. 362, pp. 351-364, 2004.

47. H. Chen, R. Kersting, and G. C. Cho, "Terahertz imaging with nanometer resolution," Applied Physics Letters 83(15), pp. 3009-3011, 2003.

48. X. C. Zhang, "Three-dimensional terahertz wave imaging," Phil. Trans. R. Soc. Lond. A 362(1815), pp. 283298, 2004.

49. N. H. E. Weste, "Private Communication," Maquarie University, July 2004.

50. D. Turchinovich, A. Kammoun, P. Knobloch, T. Dobbertin, and M. Koch, "Flexible all-plastic mirrors for the THz range," Applied Physics Letters A, 74, pp. 291-293, 2002.

51. H. Hayakawa, N. Yoshikawa, S. Yorozu, and A. Fujimaki, "Superconducting digital electronics," Proceedings of the IEEE 92(10), pp. 1549-1563, 2004.

52. V. Gopal, E. H. Chen, E. P. Kvam, and J. M. Woodall, "Behavior of a new ordered structural dopant source in InAs/(001) GaP heterostructures," Journal of Vacuum Science and Technology B 17(4), pp. 1767-1772, 1999.

53. V. G. Veselago, "The electrodynamics of substances with simultaneously negative values of e and $\mu$," Sov. Phys. Usp. 10(4), pp. 509-514, 1968.

54. D. Wu, N. Fang, C. Sun, X. Zhang, W. Padilla, D. Basov, D. Smith, and S. Schultz, "Terahertz plasmonic high pass filter," Applied Physics Letters 83(1), pp. 201-203, 2003. 